II Congresso Brasileiro de

Fluidodinâmica Computacional

27 a 29 de Junho de 2018

Rio de Janeiro, RJ

\title{
FINITE ELEMENT MESH MULTIPLICATION WITH BOUNDARY SMOOTHING
}

\author{
R. M. SILVA ${ }^{1}$, R. N. ELIAS ${ }^{2}$, A. L. G. A. COUTINHO ${ }^{3}$ \\ 1,2,3 Universidade Federal do Rio de Janeiro - UFRJ, Instituto Alberto Luiz Coimbra de \\ Pós-Graduação e Pesquisa em Engenharia - COPPE, Programa de Engenharia Civil - \\ PEC, Núcleo Avançado de Computacao de Alto Desempenho - NACAD. \\ Contact: ${ }^{1}$ romulo.silva@nacad.ufrj.br, ${ }^{2}$ renato@nacad.ufrj.br, \\ 3 alvaro@nacad.ufrj.br
}

\begin{abstract}
A procedure to recover the geometry boundary of refined finite element meshes is presented. Initially, the coarse mesh is submitted to an uniform refinement by splitting the elements, but maintaining the coarse boundary. Then, the boundary nodes of the refined mesh are filtered. To avoid search overheads, the boundary nodes and the STL vertexes are subdivided into sub-regions called BINs, and the search for closest nodes is performed only to the nodes in the same sub-region. The capability of the proposed procedure is illustrated on an example.
\end{abstract}

\section{INTRODUCTION}

In the last decades, the Finite Element Method (FEM) has become a powerful method to solve PDEs. Tools for efficient and automatic mesh generation are important prerequisites to start using FEM to do simulations according to Rypl and Bittnar (2004). However, standard commercial mesh generators are generally designed to provide piecewise linear (straight-sided tetrahedra) approximations of the geometries for simulation, and are not able to represent accurately curved geometries (Gargallo-Peiró et al., 2017).

The proposed procedure consists in a way to recover the boundary of the original geometry provided by a STL file. Initially, the mesh is submitted to an uniform refinement using the Mesh Multiplication Method (section 2.2.). So, the boundary nodes of the refined mesh are filtered and subdivided into sub-regions called BINs (section 2.3.). As well as the refined mesh nodes, the STL's vertexes are also sub-divided into the same sub-regions to avoid search overheads during the repositioning process.

The whole procedure was implemented inside EdgeCFD (Lins et al., 2009), improving the Mesh Multiplication feature which was already implemented.

\section{BACKGROUND}

\subsection{EdgeCFD}

EdgeCFD is a numerical solver that uses the FEM to solve incompressible unsteady Navier-Stokes equations and/or the transport equation. The formulation is based on the 
edge-based stabilized ans variational multiscale FEM for linear tetrahedra elements. This solver was developed using the Fortran 90 programming language, due to its extensibility and performance. It is possible to highlight yet, some other features like:

- Edge-based data structure.

- Support MPI, OpenMP or Hybrid parallelism.

- Dynamic deactivation for convection-difusion problems.

- PID adaptative time stepping for Navier-Stokes problems.

- Drag and lift force computations for node sets defined.

- Free surface computation through the Volume-Of-Fluid method (VOF).

\subsection{Mesh Multiplication Method}

The Mesh Multiplication Method, consists on a uniform refinement of FE meshes, which can be achieved by doing the subdivision of all elements. Starting from an initial coarse mesh, the element's subdivision can be done progressively until the desired refinement level is reached. That kind of refinement is also called $S U B_{8}$. Each triangular face of a tetrahedron $\boldsymbol{T}$ (Figure 1) is refined into four sub-triangles by connecting the midpoints of the edges (Liu and Joe, 1995).

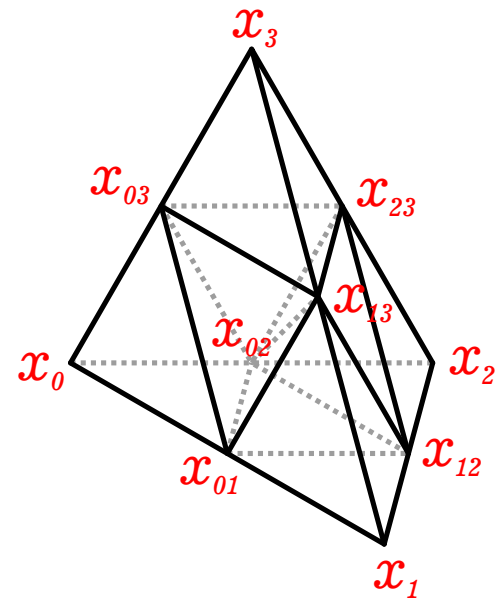

Figure 1 - Regular refinement (8-subtetrahedron subdivision); $x_{i j}=\left(x_{i}+x_{j}\right) / 2, i<j$; and the eight subtetrahedra are $\boldsymbol{T}_{\mathbf{1}}\left(x_{0}, x_{01}, x_{02}, x_{03}\right), \boldsymbol{T}_{\mathbf{2}}\left(x_{01}, x_{1}, x_{12}, x_{13}\right), \boldsymbol{T}_{\mathbf{3}}\left(x_{02}, x_{12}, x_{2}, x_{23}\right)$, $\boldsymbol{T}_{\mathbf{4}}\left(x_{03}, x_{13}, x_{23}, x_{3}\right), \boldsymbol{T}_{\mathbf{5}}\left(x_{01}, x_{13}, x_{03}, x_{02}\right), \boldsymbol{T}_{\mathbf{6}}\left(x_{01}, x_{12}, x_{13}, x_{02}\right), \boldsymbol{T}_{\mathbf{7}}\left(x_{23}, x_{02}, x_{12}, x_{13}\right)$, and $\boldsymbol{T}_{\mathbf{8}}\left(x_{23}, x_{03}, x_{02}, x_{13}\right)$ (Liu and Joe, 1995). 


\subsection{BINs and Node Repositioning}

The search for closest points does not need to be performed for points in distant regions on the domain. So, Figure 2 shows one simplest way to reduced the search overhead: a subdivision of the spatial domain using BINs (Lohner, 2004) to classify the data inside a regular mesh of bricks with nsubx $\times$ nsuby $\times$ nsubz subdivisions.

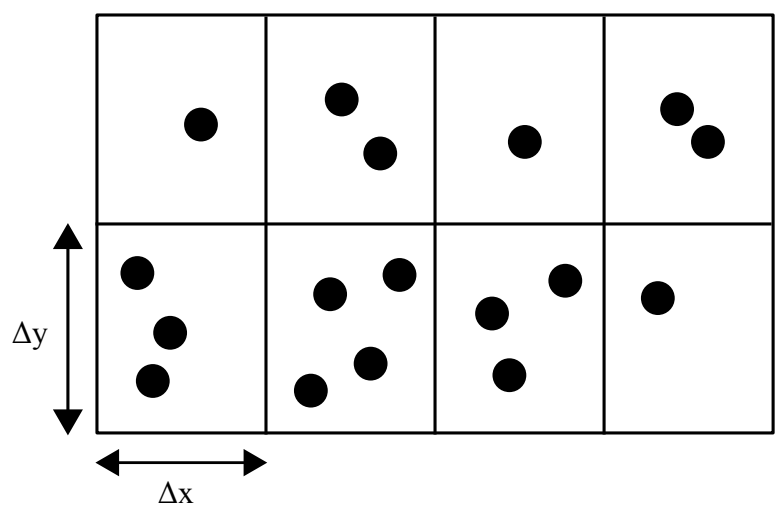

Figure $2-$ BINs

The data produced after the domain subdivision is stored into two arrays: 1 bin 1 ( 1 :npoin) and 1 bin2 (1:nbins+1), where npoin and nbins are the number of points and BINs, respectively. The lbin1 array stores the point's IDs, which are already ordered according to the bin into each point falls, and the lbin2 array indicates the BINs for each interval of points inside the lbin1 array. The criteria to do the repositioning is the Euclidian distance between the nodes on the mesh surface and the STL vertexes.

\section{TESTCASE - AN AIRFOIL IMMERSED IN A FLUID DO- MAIN}

Figure 3 shows the coarse mesh, the refined STL, and the smoothed mesh, as well as its comparison with the non-smoothed refined mesh for a case of an Fauvel 14\% Airfoil immersed in a fluid domain. Figure 3 a gives an acceptable representation of the geometry. However, after an uniform refinement, it is necessary to improve the geometry boundary targeting a refined STL file Figure 3b. The refined and smoothed geometry is presented in Figure 3c. Figure 3d shows the configuration without the boundary smoothing (represented only by edges) and the smoothed surface (represented in orange by surface with edges). The chosen metric to measure the quality of the approximation is the volume. Table 1, shows the relative error for the surface volume, taking the refined STL surface as reference. It is important to highlight that the quality of the elements closest to the boundary was not measured.

Future improvements of the procedure include a parallel implementation and the use of a metric to measure the quality of the created elements. 
Table 1 - Relative error of the volume

\begin{tabular}{|cc|}
\hline Configuration & $\epsilon=100 \cdot\left|V_{S T L}-V\right| / V_{S T L}(\%)$ \\
\hline Coarse Mesh & 0.421 \\
Non-smoothed Refined Mesh & 0.389 \\
Smoothed Refined Mesh & 0.113 \\
\hline
\end{tabular}

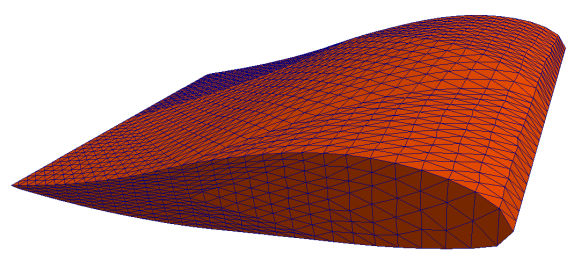

(a) Coarse Mesh

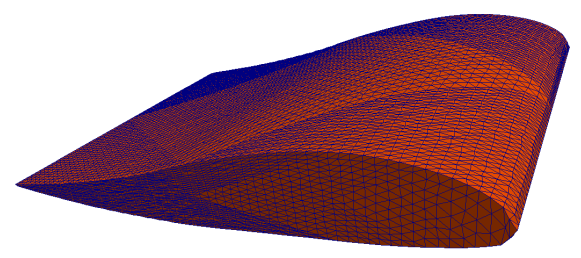

(c) Refined and Smoothed Boundary

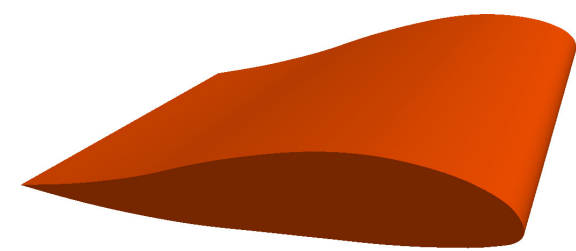

(b) Refined STL Surface

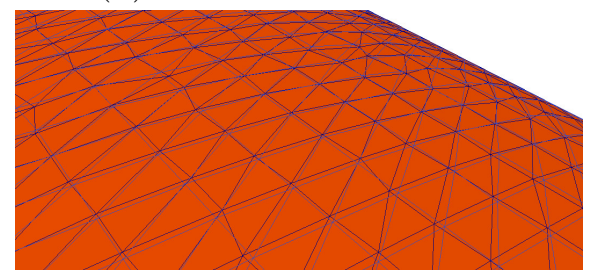

(d) Repositioning detail

Figure 3 - Stages and Components of Mesh Multiplication with Boundary Smoothing

\section{REFERENCES}

Gargallo-Peiró, A.; Houzeaux, G.; RocA, X. Subdividing triangular and quadrilateral meshes in parallel to approximate curved geometries. Procedia Engineering, 203, 310 - 322. 26th International Meshing Roundtable, IMR26, 18-21 September 2017, Barcelona, Spain, 2017.

Lins, E. F.; Elias, R. N.; Guerra, G. M.; Rochinha, F. A.; Coutinho, A. L. G. A. Edge-based finite element implementation of the residual-based variational multiscale method. International Journal for Numerical Methods in Fluids, 61(1), 1-22, 2009.

Liu, A.; Joe, B. Quality Local Refinement of Tetrahedral Meshes Based on Bisection. SIAM Journal on Scientific Computing, 16(6), 1269-1291, 1995.

Lohner, R. Applied Computational Fluid Dynamics Techniques: An Introduction Based on Finite Element Methods, volume 508, 2004.

Rypl, D.; Bittnar, Z. Triangulation of 3D Surfaces Recovered from STL Grids. Acta Polytechnica, 44(5), 61-67, 2004. 